\title{
Genistein Modulation of Immune-Associated Genes in LNCaP Prostate Cancer Cell Line
}

\author{
K. Merchant ${ }^{1, *}$, J. Kumi-Diaka ${ }^{2}$, A. Rathinavelu ${ }^{3}$, N. Esiobu ${ }^{2}$, R. Zoeller ${ }^{4}$ and V. Hormann ${ }^{2}$ \\ ${ }^{I}$ Natural Sciences Department, Albany State University, 504 College Drive, Albany GA, 31707, USA \\ ${ }^{2}$ Department of Biological Sciences, Florida Atlantic University, 3200 College Ave Davie FL 33314, USA \\ ${ }^{3}$ Rumbaugh Goodwin Institute for Cancer Research, Nova Southeastern University, Plantation Fl 33322, USA \\ ${ }^{4}$ Department of Health \& Exercise Sci, Florida Atlantic University, 3200 College Ave Davie FL 33314, USA
}

\begin{abstract}
Background: Prostate cancer is the most common form of non-dermatologic cancer and the second leading cause of cancer deaths in the United States. Survival rate for the advanced disease still remains low, so current research is aimed at alternative or adjuvant treatments that will target components of the signal pathways in the progression of carcinogenesis with little or no cytotoxicity. In this study we investigated the effect of genistein on expression levels of genes involved in the immune response pathways. The mechanism of genistein-induced cell death was also investigated. The chemosensitivity of the LNCaP prostate cancer cells to genistein was investigated using ATP and MTS assays, and a caspase binding assay was used to determine apoptosis induction. Several molecular targets/genes were determined using cDNA microarray and RT-PCR analysis.

Results: The overall data revealed that genistein induces cell death in a time- and dose-dependent manner, and regulates expression levels of several genes involved in carcinogenesis and immunity including MHC genes that are involved in immune recognition of cells and the DefB1 and the HLA membrane receptor genes involved in immunogenicity.

Conclusion: The results of the study indicate that genistein inhibits carcinogenesis of LNCaP prostate cancer growth via regulation of the identified specific targets/pathways in immunogenicity/immune response. The results thus provide significant insight into the roles that genistein could play in immune response to prostate cancer proliferation and potential role in immunotherapy and/or adjuvant therapeutic regimen.
\end{abstract}

Keywords: Genistein isoflavone, Prostate cancer, Immune-associated genes, Immunotherapeutic significance.

\section{INTRODUCTION}

Cancer is among the leading causes of death worldwide, representing $13 \%$ of all causes of mortality [1,2]. According to the WHO's estimate, the number of annual cancer deaths will rise to about 12 million in the year 2030 [1]. Prostate cancer is the sixth most commonly diagnosed cancer in the world [2]. It is the most common form of non-dermatological cancer and the second leading cause of cancer deaths in the United States [2]. ACS estimates that 1 in 6 men will be diagnosed within their lifetime and about 1 in 36 men will die from this disease [3]. The five year survival rate is almost $100 \%$ and the ten year survival rate is $95 \%$ for the local disease, however for the advanced disease, the survival rate is only $31 \%$ [2-4]. Identified risk factors that may enhance progression of this disease include age: race, family history, diet and lifestyle, environmental factors and geographic location [5-8]. Increases in relative survival rates for the localized disease may partly be due to new diagnostic tools and/or improvement in diagnostic methods. Testing and identification of new biologic markers will lead to early diagnosis and treatment of the early-stage disease [9].

*Address correcpondence to this author at the Natural Sciences Department, Albany State University, Albany, GA 31705, USA; Tel: 229-430-4823;

Fax: 229-430-4765; Email: kendra.merchant@asurams.edu
Despite the expanding knowledge of cancer genomics and high level diagnostic technology, cancer still remains a serious threat to mankind. Standard treatment regimens are still flawed with serious side effects in patients. Current research is focusing on therapeutic options with significantly reduced/minimal cytotoxicity. This includes a focus on nutrient phytochemicals which may have preventive and/or therapeutic capabilities. The apoptotic and cell cycle signaling pathways provide many avenues for researchers in searching for specific molecular targets for potential therapeutic molecules, including phytochemicals $[9,10]$.

Phytochemicals are low molecular weight, biologically active secondary plant metabolites, which have the potential to reduce the risk of certain chronic diseases such as cancer [11]. The goal of phytochemical research is to identify natural small molecules that could regulate some of the signaling pathways in carcinogenesis, leading to growth inhibition and/or cancer cell death, and eventual decrease in the mobility and mortality rates. The signaling targets aimed at include; signal transduction pathways, cell cycle regulatory molecules, angiogenesis pathways, apoptosis pathways and growth factors. A major phytochemical that has been shown to have chemopreventive properties is genistein isoflavone. 
Isoflavones are the most common form of phytoestrogens found in a variety of plants, mostly in soy [12]. Genistein (4', $5^{\prime}$, 7-trihydroxyiso-flavone) is the major bioactive isoflavone, demonstrating a variety of anti-cancer properties including: induction of apoptosis [13, 14], inhibition of tyrosine kinases and DNA topoisomerase, inhibition of angiogenesis $[11,15]$.

Genistein has also been shown to significantly regulate expression changes in genes that are involved in signaling pathways, apoptosis, the cell cycle and immune regulation [16-19]. Several studies have demonstrated immunosuppressive activities of genistein isoflavone. Genistein treatment induced a dose-dependent increase in cytotoxic T-cells activity in vivo and significant enhancement of IL-2 stimulated NK cell activity in vitro [20]. Furthermore, the proliferative response of MCF-7 breast cancer cells to genistein isoflavone in vitro has been reported [21]. The difficulties in understanding and interpreting immune responses to antigenic challenges are in part, due to lack of the functional and signaling pathway annotations of many of the key genes and gene products associated with carcinogenesis. To further understand the genetic factors in prostate cancer carcinogenesis, and the molecular mechanisms underlying the effects of genistein on LNCaP prostate cancer cells, we examined the genistein-induced expression patterns of genes in the apoptosis signaling pathway, and the potential influence on the immune-mediated pathways.

\section{MATERIALS AND METHODS}

\section{Materials/Reagents}

LNCaP prostate cancer cells (ATTC Manassas, Virginia USA); Genistein isoflavone (Sigma-Aldrich, St. Louis, Missouri, U.S.A); RPMI culture media and reagents (Invitrogen, Carlsbad, CA, USA); Dimethylsulfoxide (DMSO) (SigmaAldrich, St. Louis, MO, U.S.A).

\section{Cell Culture and Treatments}

LNCaP cells were treated in quadruplicate with a range of genistein concentrations $\left(0-100 \mu \mathrm{M} ; \mathrm{Gn}_{0-100}\right)$ or vehicle dimethylsulfoxide (DMSO) (final concentration, 0.025\%) and incubated for $48 \mathrm{hr}$; and proliferation assessed using the MTS and ATP assays/methods as described previously [22]. Results were expressed on a relative proliferation index scale (mean $\pm \mathrm{SD}$ ). Furthermore, LNCaP cells were cultured and treated with genistein for assessment and analysis of treatment-induced apoptosis and caspase protease expression as described below.

\section{MTS Assay}

LNCaP cells were plated in quadruplicate at a density of $5 \times 10^{4}$ cells per well in a 96-well MTP and treated with genistein as described above. After 24 and 48hrs incubation, 10 $\mu 1$ of MTS reagent was added to each well and incubated for $4 \mathrm{hrs}$ at $37^{\circ} \mathrm{C}$ and $5 \% \mathrm{CO}_{2}$. Absorbance of the color generated was then read at $490 \mathrm{~nm}$ using a Multiscan ELISA micro-plate reader. Graphs of the absorbance/optical density (OD) (y-axis) against the concentrations of genistein ( $\mathrm{x}$-axis) were drawn; and \% inhibition calculated against the control cells without genistein treatment.

\section{ATP Assay}

LNCaP cells were plated in quadruplicate at a density of $5 \times 10^{4}$ cells per well in a 96-well MTP and treated with genistein as described above. After 24 and $48 \mathrm{hrs}$ incubation, 50 $\mu \mathrm{l}$ of lysing solution was added to each well and incubated for five minutes; followed by addition of $50 \mu \mathrm{l}$ of luciferin/ luci-ferase solution to each well. Luminescence was determined after 5 min using a luminometer. ATP concentration was determined by comparing the luminescent values against a standard curve, from which \% inhibition was calculated.

\section{Caspase Protease Analysis}

The role of caspase- 3 in genistein-induced apoptosis in LNCaP was assessed with a caspase- 3 colorimetric activity assay. Briefly, 2- 4 × $10^{6}$ cells/well were cultured and treated with various concentrations of genistein $\left(\mathrm{Gn}_{0-100}\right)$ or with topotecan $(50 \mu \mathrm{M})$ as a positive inducer of apoptosis [22]. The samples were treated both in the presence and absence of a caspase inhibitor (Z-DEVD-FMK). The cells were harvested after 24 and 48 hrs incubation then centrifuged (1500 $\mathrm{rpm}$ for $5 \mathrm{~min}$ ), re-suspended in $200 \mu \mathrm{l}$ of lysis buffer and incubated on ice for $20 \mathrm{~min}$. The suspensions were again centrifuged for $5 \mathrm{~min}$ and the supernatant collected for protein analysis, using the BCA protein assay kit (Pierce) to determine the protein concentration of each sample. $30 \mu \mathrm{g}$ of protein lysate of each sample was then assayed for caspase activity. The assay involves caspase mediated cleavage of a labeled peptide substrate, acetyl-Asp-Glu-Val-Asp pnitroanilide (Ac-DEVD- $p \mathrm{NA})$. The $p$-nitroaniline ( $p \mathrm{NA}$ ) product was quantified using a plate reader at $405 \mathrm{~nm}$, giving a direct indication of caspase- 3 activity. Fold-increase in caspase- 3 activity was then calculated by comparing the absorbance values of the treated samples to that of the control sample.

\section{cDNA Microarray Analysis}

LNCaP cells were cultured and treated with $50 \mu \mathrm{M}$ genistein $\left(\mathrm{Gn}_{0-100}\right)$ [22] after which RNA was extracted using the RNeasy mini kit (Qiagen) according to the manufacturer's protocol. The RNA samples were submitted to Genomics Core (Scripps Research Institute, Jupiter, FL) for microarray analysis. Briefly, RNA samples were quantified using the Nano-Drop ND-1000 protocol. Double-stranded cDNA was prepared from $1 \mu \mathrm{g}$ of total RNA using the Affymetrix cDNA synthesis kit and then in vitro transcribed using an IVT labeling kit (Affymetrix) with the cRNA product purified using a GeneChip Sample Cleanup Module (Affymetrix). $20 \mu \mathrm{g}$ biotin-labeled cRNA was fragmented and hybridized to Affymetrix Human Gene chip U133 plus 2.0 arrays overnight in the Affy 640 hybridization oven with a speed of $60 \mathrm{rpm}$ for $16 \mathrm{hr}$. Microarrays were washed and stained using the Affymetrix Fluidics Station FS400.

GeneChip arrays were scanned using a GeneChip Scanner 3000 (Affymetrix). The probe set intensities were quantified using the GeneChip Operating Software (GCOS) and analyzed with GCRMA normalization using Array Assist Software (Stratagene, La Jolla, CA). All hybridized chips met standard quality control criteria, and mean fluorescence values of each array were scaled to a mean intensity of 500 . 
Gene expression of the treated cells was compared with control (untreated) cells to determine changes in expression of target genes. Functional cluster analysis was done using the Database for Annotation, Visualization and Integrated Discovery (DAVID) [25]. Results of the microarray analysis were verified by RT-PCR as described below.

\section{RT-PCR Analysis}

The RNA extracted from the cell samples was subjected to RT-PCR analysis using the Access RT-PCR System (Promega). Selected genes were reverse transcribed to verify the results of the array gene expression changes. The Access RT-PCR System is designed for convenient and sensitive detection and analysis of RNA by one-step RT-PCR. cDNA synthesis and PCR are performed in a single tube using gene specific primers $(4 \mu \mathrm{M})$ and $1 \mu \mathrm{g}$ of total RNA. The specific primers used were bax, bcl-x, vegf, mdm-2 and beta-actin as an internal loading control $[23,24]$. The amplified products were separated on a $1 \%$ agarose gel stained with ethidium bromide. The results of this experiment were compared to that of the microarray analysis to determine if similar changes in gene expression were observed in target genes.

\section{STATISTICAL ANALYSIS}

Experiments were performed in duplicates to confirm similar results. Significance of the differences in mean values was determined using the Student's t-test and $P$ value of $\leq 0.05$ was considered statistically significant.

\section{RESULTS}

\section{Genistein-induced Growth Inhibition}

The data from both MTS and ATP assays show that genistein inhibited cell growth by inducing concentration- and time-dependent cell death (Figs. 1 and 2). Being a more sensitive assay, the ATP assay revealed progressively significant growth inhibition of the cells from 0 to $60 \mu \mathrm{M}$ genistein $(\mathrm{p}<0.05)$ after just $24 \mathrm{hr}$ of treatment. After $48 \mathrm{hr}$, a similar pattern of growth inhibition was seen. Above $60 \mu \mathrm{M}$, there

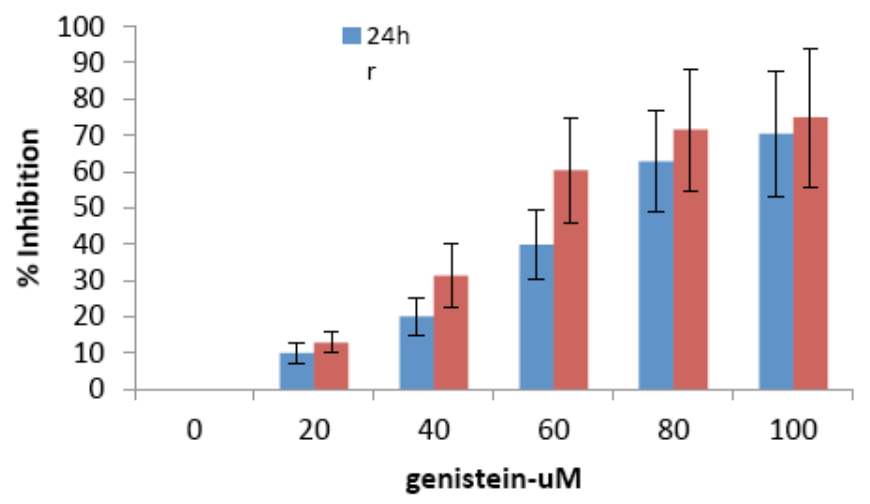

Fig. (1). Determination of cytotoxic effect of genistein on growth inhibition of LNCaP cells using the ATP assay. Cells were treated with various concentrations of genistein and \% inhibition was determined using the ATP assay. Data are representative of two independent experiments and are expressed as the mean \pm STDV. Bar $=$ STDV. Significant data is marked with an asterisk. was no significant difference seen between genistein-induced cell death at 24 and 48 hrs after treatment (p > 0.05) (Fig. 1). The MTS assay showed obvious significant growth inhibition at 24 and $48 \mathrm{hrs}$ from 0 to $50 \mu \mathrm{M}$ of genistein $(\mathrm{p}<0.05)$ (Fig. 2). Above $50 \mu \mathrm{M}$, there was a significant seen in growth inhibition induced at both time periods, indicating that the effect of genistein is both time- and dose-dependent. Based on these preliminary findings, a smaller range of genistein was used to carry out further studies.

\section{Caspase Protease Analysis}

The results showed that genistein induced caspase-3 activity which was time- and dose-dependent (Fig. 3). This correlates with the results of the ATP and MTS assays. In the presence of caspase- 3 inhibitor, caspase- 3 induction/activity was the same after $24 \mathrm{hrs}$ even at high concentrations of genistein. However, in the absence of any inhibition there was a 1.5 fold increase in caspase activity at $50 \mu \mathrm{M}$ genistein,

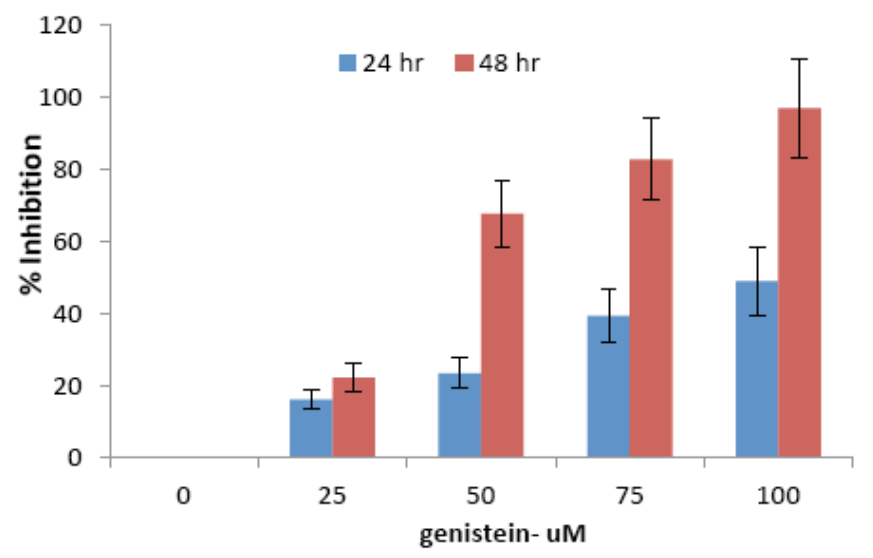

Fig. (2). Determination of the cytotoxic effect of genistein on growth inhibition of LNCaP cells using the MTS assay. Cells were treated with various concentrations of genistein and \% inhibition was determined using the MTS assay. Data are representative of two independent experiments and are expressed as the mean \pm STDV. Bar $=$ STDV. Significant data is marked with an asterisk.

Fold increase in caspase- 3 activity

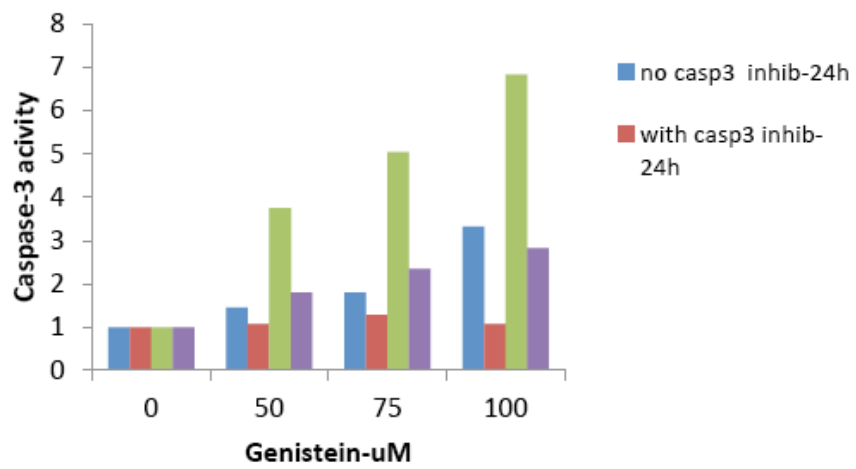

Fig. (3). Effect of genistein treatment on caspase-3 activity in LNCaP cells after 24 hrs. Cells were treated and changes in caspase-3 activity were determined. Data are representative of two independent experiments and are expressed as the mean \pm STDV. Bar $=$ STDV. Significant data is marked with an asterisk. 




Fig. (4). Effect of genistein treatment on gene expression levels of selected genes in LNCaP cells after $48 \mathrm{hrs}$. Cells were treated with $50 \mu \mathrm{M}$ of genistein and gene expression levels were determined using Access RT-PCR system.

after just 24 hrs, and almost a 4-fold increase at the same concentration after $48 \mathrm{hrs}$ (Fig. 3). These results indicate that genistein-induced cell death involves the activation of the caspase pathway which is involved in apoptosis.

\section{Microarray and RT-PCR Analysis}

The effect of genistein on gene expression in LNCaP cells was investigated using microarray and RT-PCR analysis. LNCaP cells were treated with $50 \mu \mathrm{M}$ genistein because previous studies have indicated that genistein inhibition is dose-dependent [22, 27-29] and our MTS and ATP data indicated that inhibition is not significant above $60 \mu \mathrm{M}$.

The Affymetrix human gene chip U133 plus 2.0 array was used to compare expression of genes in genistein treated and untreated LNCaP cells. A 2-fold difference in expression, compared to the control, was considered significant. A functional annotation cluster analysis of all these genes was done and changes in specific pathways were investigated based on KEGG annotations [26]. A number of pathways were found to be regulated by the treatment. Exposure of LNCaP cells to genistein treatment resulted in up-regulation and down-regulation of a number of genes that play a role in immune response within the body, in addition to other general genes (Tables $\mathbf{1}$ and $\mathbf{2}$ ).

Up-regulated genes having a10-fold or higher differential change in expression levels were also analyzed further and clustered according to function. Neu1 (sialidase1), Insig1, GPNMB and CD 68 which were up-regulated are genes that play a role in immune recognition of cancer cells. Another group of genes affected by genistein include the MHC proteins (major histocompatibility complex) which are cell surface antigens involved in immune recognition of cells. DefB1 expression was also up-regulated (28.8 fold-change) by genistein. Other genes that were affected include members of the TNF super-family which are involved in the apoptotic pathway. BrunoL6 gene was not included in the cluster, but this gene was the most up-regulated gene, showing a fold-change of 116.0 .

Table 1. Apoptosis-regulated Genes Showing Differential Expression after Treatment with Genistein

\begin{tabular}{|c|c|c|}
\hline \multicolumn{3}{|c|}{ Down-Regulated Genes } \\
\hline Bcl-2 & B-cell CLL/Lymphoma 2 & 4.8 \\
\hline Bcl-X & Bcl-2 like-1 & 3.0 \\
\hline BCL11A & B-cell CLL/ Lymphoma 11a (zinc finger protein) & 2.1 \\
\hline Fas & TNF receptor superfamily member 6 & 4.0 \\
\hline TNFSF9 & Tumor necrosis factor (ligand) superfamily member 9 & 2.9 \\
\hline BAM/BIM/Bcl2L11 & Bcl-2 like-11 & 6.9 \\
\hline TNFSF9 & Tumor necrosis factor (ligand) superfamily member 9 & 2.9 \\
\hline Mdm-2 & Double minute 2 protein & 3.7 \\
\hline GPNMB & glycoprotein (transmembrane) $\mathrm{nmb}$ - tumor associated antigen & 26.3 \\
\hline BrunoL6 & $\begin{array}{l}\text { Bruno-like } 6 \text { RNA binding protein - involved in regulation of pre-mRNA alternative splicing } \\
\text { and mRNA editing and translation }\end{array}$ & 116.0 \\
\hline
\end{tabular}


Table 2. Immune-system Associated Genes Showing Differential Expression after Treatment with Genistein

\begin{tabular}{|l|l|l|}
\hline \multicolumn{2}{|c|}{ Up-regulated Genes } & \multicolumn{1}{|c|}{ Gene Name } \\
\hline \hline HLene Symbol & & 3.0 \\
\hline HLA-G & Major histocompatibility complex class I, E & 2.2 \\
\hline HLA-B & Major histocompatibility complex class I, G & 2.0 \\
\hline HLA-C & Major histocompatibility complex class I, B & 2.0 \\
\hline HLA-DMA & Major histocompatibility complex class I, C & 2.3 \\
\hline ICOSLG & Major histocompatibility complex class II, DM alpha & 2.5 \\
\hline PDGFA & Inducible T-cell co-stimulator ligand & 2.0 \\
\hline Insig 1 & Platelet-derived growth factor alpha polypeptide & 10.1 \\
\hline NEU1 & Insulin induced gene 1 - ER membrane protein that plays a role in regulating cholesterol levels in cell & 12.1 \\
\hline CD68 & Sialidase I (lysosomal sialidase) - important for function of macrophages & 12.1 \\
\hline DEFB1 & CD68 molecule-plays a role in phagocytic activity of macrophages & 28.8 \\
\hline
\end{tabular}

Based on our preliminary findings and previous studies [22], a few genes were further selected and subjected to RTPCR analysis to confirm the results of the microarray analysis. These include Bax, Bcl-2, Bcl-x, VEGF, mdm-2, and $\beta$ actin (as a loading control). The results indicate that mdm-2 showed an increase in expression when cells were treated with genistein; which correlates with the microarray results. The anti-apoptotic proteins $\mathrm{Bcl}-2$ and $\mathrm{Bcl}-\mathrm{x}$ were downregulated whereas VEGF and Bax were slightly but insignificantly up-regulated by genistein ( $p>0.05)$. The PCR results obtained were consistent with the microarray data obtained and confirm the effects of genistein on gene expression profiles within the $\mathrm{LNCaP}$ prostate cancer cell lines as outlined in Tables $\mathbf{1}$ and $\mathbf{2}$.

\section{DISCUSSION}

\section{Growth Inhibition of LNCaP Cells}

Genistein induced a time- and dose- dependent apoptotic cell death in the LNCaP cells consistent with previous studies [22, 27, 28]. The results of the ATP and MTS proliferation assays are consistent with these studies and indicate the chemosensitivity of LNCaP prostate cancer cells towards genistein. Caspase-3 activity was also increased after exposure to genistein. Caspase- 3 is an effector caspase and plays a central role in apoptotic cell death, so detection of this caspase indicates activity of other up-stream caspases. Once activated, caspase-3 has been shown to initiate fragmentation of DNA which ultimately leads to cell death $[29,30]$.

\section{Modulation of Genes Involved in Immune Response}

Tumor metastasis is a complex process consisting of multiple steps regulated by tumor-associated factors and signaling pathways, including expression of bcl-2 protooncogenes. Genistein seems to have pleiotropic actions, one of which involves the Bcl-2 gene or protein. In this study, the anti-apoptotic proteins, Bcl-2 and Bcl-x were downregulated, and the pro-apoptotic protein Bax was slightly but insignificantly up-regulated by genistein; a finding which is consistent with previous studies. Previous studies reported the impact of bcl2-family proteins on immunity, where radiation-induced apoptosis was blocked by bcl-x upregulation; and memory $\mathrm{T}$ cells remain resistant to apoptosis due to (contact with antigen-bearing APC and IL-2R occupancy result in the) the expression of the bcl-2 and bcl-x anti-apoptosis proteins. [31]. In addition, genistein-induced increase in interleukin-2 (IL-2)-stimulated natural killer (NK) cell activity was reported [20]. Furthermore, it has been reported that genistein treatment induced a dosedependent increase of cytotoxic T-lymphocyte (Tc) activity in mice. The present observations/data are consistent with the previous findings; and indicate the potential role of genistein in immunotherapy.

Genistein treatment resulted in up-regulation (and downregulation) of numerous genes involved in the immune response system. Neu1 and CD 68 were both up-regulated and are both involved in immune recognition of cells. Neu 1 is involved in differentiation of monocytes into macrophages and is important for the primary function of macrophages [32] while CD68 is an antigen marker that promotes phagocytosis and mediates recruitment and activation of macrophages. CD68 is a surface marker commonly used to identify macrophages so further studies are needed to confirm expression levels and determine the role of increased expression of this marker in prostate cancer cells.

Other groups of genes modulated by genistein include the MHC (major histocompatibility complex) and DefB1. MHC 
proteins are cell surface antigens involved in immune recognition of cells. Foreign particles or foreign proteins (antigens) are displayed on the cell surface by MHC receptors, which then present them to immune cells. Cancer cells are able to evade the immune system by mutating these receptors or masking them; and so genistein-induced upregulation of genes that code for these receptors indicates that genistein can play a significant role in activation of the immune response within the body. DefB1 expression is normally lost in and during prostate cancer carcinogenesis [33]; and it was found to be up-regulated by genistein (28.8fold change). This gene has antimicrobial activity towards bacteria and fungi and is involved in chemo-attraction of immune cells $[34,35]$. Up-regulation of this DefB1 suggests a potential phytotherapeutic-phytopreventive significance of genistein in immunotherapy. Current research trials in immunotherapy have shown that activation of immune cells such as macrophages and/or dendritic cells enables recognition of the cancer cells and leads to eradication of the tumor and, increases disease-free survival of patients [36, 37]. Such previous clinical trials add credence to the potential usefulness of genistein isoflavone, as further demonstrated in this study.

\section{CONCLUSION}

Our study provides evidence that genistein affects/regulates expression of some of the genes that are known to be involved in carcinogenesis and immunogenicity of LNCaP cells and most human tumors. Genistein treatment resulted in up-regulation and down-regulation of a number of genes that play a role in the immune response within the body. The results thus provide significant insight into the roles that genistein could play in immune response to prostate cancer proliferation and potential role in immunotherapy and/or adjuvant therapeutic regimen. A number of other molecular targets were also identified and further research will enable us to elucidate the significance of these genes and their mechanism of actions in genistein-induced apoptosis in prostate cancer cells.

\section{AUTHORS' CONTRIBUTION}

JK-D, KM and AR designed the study; JK-D, KM and $\mathrm{VH}$ collected and analyzed the data; NE and RZ were involved in data analysis and interpretation. All authors contributed to writing and review of the manuscript. None of the authors had any conflict of interest in this program.

\section{ACKNOWLEDGEMENTS}

The authors acknowledge the partial material support of Florida Atlantic University. The collaboration and material support of Rambough-Goodwin Institute for Cancer Research (RGICR) of Nova Southeastern University is highly acknowledged. We also recognize the technical and research assistance of our laboratory staff at Florida Atlantic University and RGICR

\section{CONFLICT OF INTEREST}

None declared.

\section{REFERENCES}

[1] WHO's fight against cancer: strategies that prevent cure and care Geneva, Switzerland, World Health Organization, 2007. Available from: http: //www.who.int/cancer/publicat/WHOCancerBrochure2007.FINALweb.pdf

[2] Jemal A, Siegel R, Xu J Ward E. Cancer Statistics, 2010. CA: A Cancer Journal for Clinicians 2010; 60: 277-300. Available from: http: //onlinelibrary.wiley.com/doi/10.3322/caac.20073/pdf

[3] American Cancer Society. Cancer Facts \& Figures 2011. Atlanta: American Cancer Society; 2011. Available from: http: //www. cancer.org/Research/CancerFactsFigures/CancerFactsFigures/cancerfacts-figures-2011

[4] Stanford JL, Stephenson RA, Coyle LM, et al. Prostate Cancer Trends 1973-1995, SEER Program, National Cancer Institute. NIH Pub. No. 99-4543. Bethesda, MD, 1998. Available from: http: //seer.cancer.gov/publications/prostate/

[5] Mettlin C. Recent developments in the epidemiology of prostate cancer. Eur J Cancer 1997; 33(3): 340-47.

[6] Marks LS, Kojima M, Demarzo A, et al. Prostate Cancer in Native Japanese and Japanese American Men: Effects of Dietary Differences on Prostatic Tissue. Urology 2004; 64(4): 765-71.

[7] Colli JL, Colli A. International comparisons of prostate cancer mortality rates with dietary practices and sunlight levels. Urol Oncol 2005; 23: 390-98.

[8] Klassen AC, Platz EA. What Can Geography Tell Us About Prostate Cancer? Am J Prev Med 2006; 30(2S): S7-S15.

[9] Gao X, Porter AT, Grignon DJ, Pontes JE, Honn KV. Diagnostic and Prognostic Markers for Human Prostate Cancer. Prostate 1997; 31: 264-81.

[10] Hasler CM, Blumberg JB. Symposium on Phytochemicals: Biochemistry and Physiology. Am Soc Nutrition Sci 1999; 129. 756S-757S.

[11] Sarkar FH, Yiwei L. Mechanisms of cancer chemoprevention by soy isoflavone genistein. Cancer Metastasis Rev 2002; 21: 265280.

[12] Tham DM, Gardner CD, Haskell WL. Potential health benefits of dietary phytoestrogens: a review of the clinical, epidemiological and mechanistic evidence. J Clin Endocrinol Metab 1998; 83: 2223-35.

[13] Wei H, Bowen R, Cai Q, Barnes S, Wang Y. Antioxidant and antipromotional effects of the soybean isoflavone genistein. Proc Soc Exp Biol Med 1995; 208: 124-30.

[14] Polkowski K, Mazurek AP. Biological properties of genistein. A review of in vitro and in vivo data. Acta Pol Pharm 2000; 57: 135-55.

[15] Dixon RA, Ferreira D. Genistein Phytochemistry 2002; 60; 205-11.

[16] Rice L, Handayani R, Cui Y, et al. Soy Isoflavones Exert Differential Effects on Androgen Responsive Genes in LNCaP Human Prostate Cancer Cells. J. Nutr 2007; 137: 964-72.

[17] Bato L, Steinarjohan K, Fahri S. Genistein differentially modulates androgen-responsive gene expression and activates JNK in LNCaP cells. Oncology reports 2008; 19(5): 1231-5.

[18] Bhamre S, Sahoo D, Tibshirani R, Dill DL, Brooks JD. Gene Expression Changes Induced by Genistein in the Prostate Cancer Cell Line LNCaP. Open Prostate Cancer J 2010; 3: 86-98.

[19] Takahashi Y, Lavigne JA, Hursting SD, et al. Using DNA microarray analyses to elucidate the effects of genistein in androgenresponsive prostate cancer cells: Identification of novel targets. Mol Carcinog 2004; 41: 108-19.

[20] Tai L, Guo J, McCay A, et al. Genistein Modulates Immune Responses and increases Host Resistance to B16F10 Tumor in Adult Female B6C3F1 Mice. J Nutrition 2001; 131: 3251-8.

[21] Lavigne JA, Takahashi Y, Chandramouli GV, et al. Concentrationdependent effects of genistein on global gene expression in MCF-7 breast cancer cells: an oligo microarray study. Breast Cancer Res Treat 2008; 110(1): 85-98

[22] Merchant K, Rathinavelu A, Kumi-Diaka J, et al. Molecular targets of genistein in prostate cancer cells. Functional Foods in Health and Disease 2011; 3. Available from: http: //www.functionalfoodscenter. net/The_Journal_of_FFHD.html.

[23] Zhang $\mathrm{H}$, Kim KJ, Edwards CA, Xu Z, Taichman R, Wang C. Clusterin inhibits apoptosis by interacting with activated Bax. Nature Cell Biol 2005; 7(9): 909-15. 
[24] Liu J, Yin S, Reddy N, Spencer C, Sheng S. Bax Mediates the Apoptosis-Sensitizing Effect of Maspin. Cancer Res 2004; $64 ; 1703$.

[25] Glynn D, Sherman BT, Hosack DA, et al. DAVID: Database for Annototation, Visualization and Integrated Discovery. Genome Biol 2003 4(5): P3.

[26] Kanehisa M, Goto S, Kawashima S, Nakaya A. The KEGG databases at GenomeNet. Nucleic Acids Res 2002; 30(1): 42-6.

[27] Kumi-Diaka J, Saddler-Shawnette S, Aller A, Brown J. Potential mechanism of phytochemical-induced apoptosis in human prostate adenocarcinoma cells: Therapeutic synergy of genistein and $\beta$-lapachone combination treatment. Cancer Cell Int 2004; 4: 5 .

[28] Kumi-Diaka J, Merchant K, Haces A, Hormann V, Johnson M. Genistein-Selenium combination induces growth arrest in prostate cancer cells. J Med Food 2010; 13(4): 1-9.

[29] Kumi-Diaka J, Butler A. Caspase-3 protease activation during the process of genistein- induced apoptosis in TM4 testicular cells. Biol Cell 2000; 92: 115-24

[30] Shi Y. Mechanisms of caspase activation and inhibition during apoptosis. Mol Cell 2002; 9: 459-70.

[31] Mueller DL, Seiffert S, Fang W, Behrens TW. Differential regulation of bcl-2 and bcl-x by CD3, CD28, and the IL-2 receptor in cloned CD4+ helper T-cells. A model for the long- term survival of memory cells. J Immunol 1996; 156(5): 1764-71.

[32] Liang F, Seyrantepe V, Landry K, et al. Monocyte Differentiation Up-regulates the Expression of the Lysosomal Sialidase, Neu1 and Triggers Its Targeting to the Plasma Membrane via Major Histocompatibility Complex Class II-positive Compartments. J Biol Chem 2006; 281(37): 27526-38

[33] Donald CD, Sun CQ, Lim SD, et al. Cancer specific loss of betadefensin 1 in renal and prostatic carcinomas. Lab Invest 2003; 83: 501-5.

[34] Zucht HD, Grabowsky J, Schrader M, et al. Human beta-defensin1: A urinary peptide present in variant molecular forms and its putative functional implication. Eur J Med Res 1998; 3(7): 315-23.

[35] Yang D, Chertov O, Bykovskaia SN, et al. $\beta$-defensins: linking innate and adaptive immunity through dendritic and T cell CCR6. Science 1999; 286(5439): 525-8.

[36] Simons JW, Sacks N. Granulocyte-macrophage colony-stimulating factor-transduced allogeneic cancer cellular immunotherapy: The $\mathrm{GVAX}^{\circledR}$ vaccine for prostate cancer. Urol Oncol 2006; 24(5): 419-24.

[37] Yamamoto N, Suyama H, Yamamoto N. Immunotherapy for prostate cancer with $\mathrm{Gc}$ protein-derived macrophage activating factor, GcMAF. Transl Oncol 2008; 1(2): 65-72.

(C) Merchant et al.; Licensee Bentham Open.

This is an open access article licensed under the terms of the Creative Commons Attribution Non-Commercial License (http://creativecommons.org/licenses/ by-nc/3.0/) which permits unrestricted, non-commercial use, distribution and reproduction in any medium, provided the work is properly cited. 